\title{
Traveling-wave Thomson scattering for electron-beam spectroscopy
}

\author{
Q. Chen $\odot,{ }^{1}$ V. Horný $\odot,{ }^{2}$ R. Syed $\odot,{ }^{1}$ and D. Umstadter ${ }^{1, *}$ \\ ${ }^{1}$ Department of Physics and Astronomy, University of Nebraska-Lincoln, Lincoln, Nebraska 68588, USA \\ ${ }^{2}$ Department of Physics, Chalmers University of Technology, Fysikgården 1, 41258 Gothenburg, Sweden
}

(Received 31 May 2020; accepted 26 February 2021; published 15 March 2021)

\begin{abstract}
We propose a method to use traveling-wave Thomson scattering for spatiotemporally-resolved electron spectroscopy. This can enable ultrafast time-resolved measurements of the dynamics of relativistic electrons in the presence of extremely intense light fields, either in vacuum or in plasma, such as in laser wakefield accelerators. We demonstrate, with test-particle simulation and analysis, the capability of this technique for measurements of various high field phenomena: radiation reaction of electrons due to scattering, dephasing of a laser wakefield accelerator, and acceleration of electrons in multiple buckets by a laser wakefield.
\end{abstract}

DOI: 10.1103/PhysRevAccelBeams.24.032901

\section{INTRODUCTION}

Relativistic electron beams provide the foundation for accelerator-driven $x$-ray light sources, including synchrotrons [1], free-electron lasers [2] and Compton sources [3]. A variety of methods have been developed to diagnose electron beam qualities. For instance, dipole magnet spectrometers are in standard use to measure electron energy. However, accurate measurement of multi-GeV electrons requires large and expensive magnets, the amount of spectral information is limited, and the measurement itself necessarily perturbs the electron beam under scrutiny.

An alternative electron-energy diagnostic is Thomson scattering (TS), also referred to as inverse Compton scattering (ICS). When laser light is TS by a relativistic electron, the energy of the scattering photon is Dopplerupshifted by an amount proportional to the electron energy. This dependence allows the electron energy to be measured simply by measuring the energy of the scattered photon, as was demonstrated experimentally over a large energy range [4,5]. TS has also been shown to be useful in diagnosing other electron beam parameters, such as energy spread and beam emittance [6-9]. Also, because the energy of the electron changes only negligibly after scattering, electron spectroscopy with TS is a noninvasive diagnostic. Moreover, it is quite convenient to use TS spectroscopy to diagnose electron beams that are either driven by laserdriven accelerators or used in ICS x-ray light sources [10], because the same laser can drive them all.

*donald.umstadter@unl.edu

Published by the American Physical Society under the terms of the Creative Commons Attribution 4.0 International license. Further distribution of this work must maintain attribution to the author(s) and the published article's title, journal citation, and DOI.
Accurate spectroscopy with TS must satisfy two requirements: (1) high TS conversion efficiency, and (2) a spectral shift that depends primarily on electron energy, rather than on the detailed characteristics of the laser pulse, such as its intensity and pulse duration. However, it is challenging to satisfy both these requirements simultaneously, at least with typical Gaussian laser-beam focusing, due to the physics of TS (discussed in detail below). To achieve high conversion efficiency, the scattering laser pulse must be focused to high intensity, which causes a redshift as well as a broadening of the TS spectrum [11]. Additional spectral broadening results when ultrashort-duration laser pulses are used to reach high-intensity because the electrons undergo only very few oscillations during TS in this case [12].

We propose a method to circumvent these drawbacks by replacing the usual Gaussian beam focusing with travelling-wave focusing. In this case, an obliquely propagating ultrashort laser pulse with pulse-front-tilt co-moves with the electrons. The TS spectrum then does not become broadened and depends primarily on the electron energy, not the laser intensity or pulse duration. The reasons are two-fold: (1) the laser intensity is low (because the focal spot size can be large), and (2) the number of electron oscillations is large (a thousand-fold larger than with Gaussian focusing) because the laser pulse moves colinearly along with the electrons. This geometry for TS is commonly referred to as traveling-wave Thomson scattering (TWTS) $[12,13]$. Our concept to use TWTS as a magnet-free spatiotemporally resolved spectrometer for relativistic electrons is also advantageous as compared to the conventional Thomson scattering spectrometers because of the spatiotemporal locking between the laser pulse and the electron beam. As a result, and as discussed in this paper, the TWTS spectrum becomes a dynamic diagnostic for various spatiotemporal processes, such as electron-energy loss due to radiation reaction, and the 
acceleration-dephasing length or the electron-bunch profile in a laser-wakefield accelerator (LWFA).

It should be noted that TWTS has been proposed as highefficiency and narrow-bandwidth ICS x-ray sources, as well as for electron bunching in optical free electron lasers [14]. To achieve TWTS, particular arrangements of compressor gratings have been designed for accurate dispersion control. The traveling-wave geometry has also been proposed for several other applications, including $\mathrm{THz}$ generation [15], ultrafast electron diffraction [16], traveling-wave excitation of x-ray lasers [17-19], and plasma based Raman amplification and compression [20,21].

This paper is organized as follows. In Sec. II, a brief model description is provided; in Sec. III, simulation results on three applications are presented; and in Secs. IV and V, a discussion and summary are found.

\section{MODEL DESCRIPTION}

The two-dimensional simulation model consists of two parts: a test-particle model and a radiation model. The relativistic test-particle model simulates the motions of single electrons inside the high-intensity laser fields:

$$
\begin{gathered}
\frac{d \mathbf{p}}{d t}=\mathbf{F}_{L}+\mathbf{F}_{R R} \\
\mathbf{p}=\gamma(\mathbf{v}) m_{e} \mathbf{v} \\
\mathbf{F}_{L}=e(\mathbf{E}+\mathbf{v} \times \mathbf{B}) \\
\mathbf{F}_{R R}=\frac{2 e^{3}}{3 m_{e} c^{3}} \dot{\mathbf{E}}+\frac{2 e^{4}}{3 m_{e}^{2} c^{4}} \mathbf{E} \times \mathbf{H}
\end{gathered}
$$

where $\mathbf{p}$ is the electron momentum, $\mathbf{F}_{L}$ is the Lorentz force and $\mathbf{F}_{R R}$ is the radiation reaction force expressed by the Lorentz-Abraham-Dirac (LAD) equation [22]; $\gamma=$ $1 / \sqrt{1-v^{2} / c^{2}}$ is the Lorentz factor, $v$ is the electron velocity, $c$ is light velocity in vacuum, $e$ is the electron charge, $m_{e}$ is the electron rest mass, $\mathbf{E}$ is the electric field, $\mathbf{B}$ is the magnetic field, $\mathbf{H}$ is the magnetizing field.

Based on the electron trajectory calculated by the testparticle simulation, the radiation model simulates electron radiation, using previously demonstrated method based on Fourier transformations [23].

The simulation layout is briefly sketched in Fig. 1(a). The right propagating electron spatiotemporally overlaps with an obliquely propagating and pulse-front titled laser pulse. The overlap is achieved and maintained when the electron-laser crossing angle $\phi$ and the pulse-front tilt angle $\alpha$ satisfy [12]:

$$
\alpha=\arctan \left(\frac{1-\beta \cos \phi}{\beta \sin \phi}\right) \stackrel{\beta \sim 1, \phi \sim 0}{\simeq} \phi / 2
$$
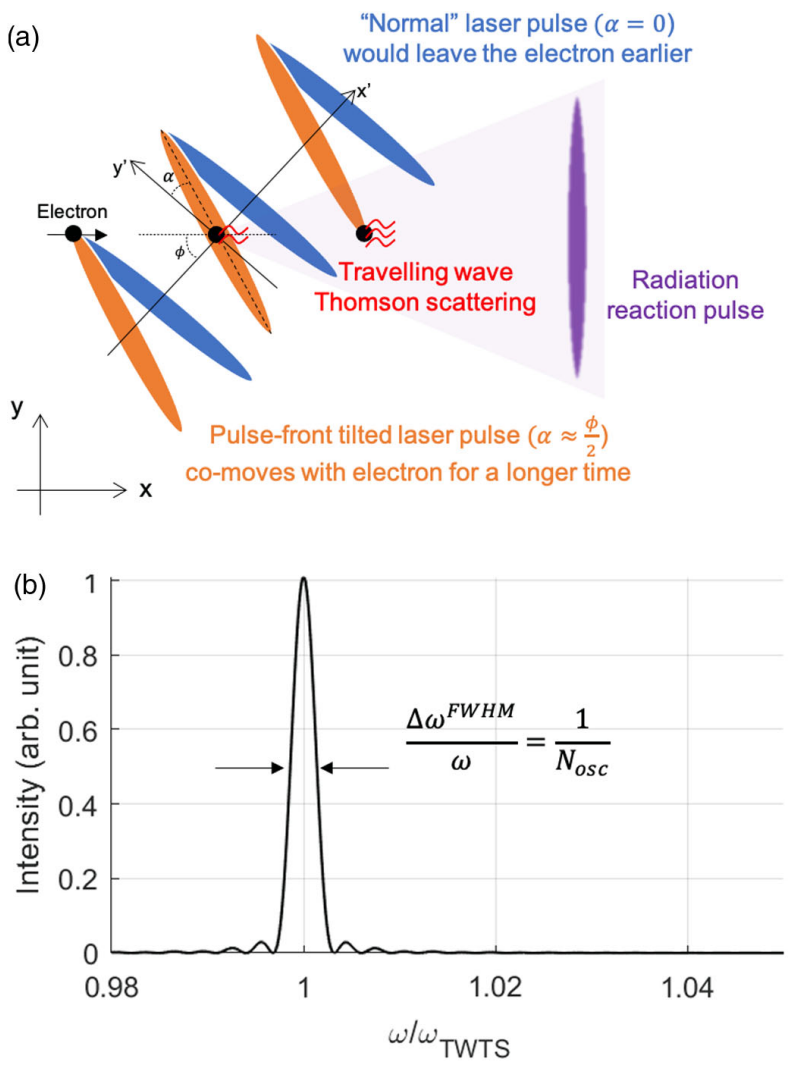

FIG. 1. (a) Geometry of TWTS, for which the pulse-front tilted laser pulse is spatiotemporally locked with the relativistic electron. The normal pulse, without pulse-front tilt, slips out of the electron after a short distance of propagation. Another pulse can be added, for example, to investigate the radiation reaction process; (b) A typical spectrum of TWTS. Due to the small amplitude of the TWTS laser field, the central x-ray photon energy is the linear limit [Eq. (6)]. The bandwidth is inversely proportional to the number of electron oscillations in TWTS laser field, or equivalently, inverse proportional to the traveling-wave distance.

where $\beta=v / c$ is the normalized electron velocity. The fundamental $\mathrm{x}$-ray wavelength of TWTS is

$$
\omega_{\mathrm{TWTS}}=\omega_{0} \times \frac{2 \gamma_{0}^{2}(1-\beta \cos \phi)}{1+a_{0}^{2} / 2+\gamma_{0}^{2} \theta^{2}}
$$

where $\omega_{0}=2 \pi / \lambda_{0}$ is the laser angular frequency, $\lambda_{0}$ is the laser wavelength, $\gamma_{0}$ is the initial Lorentz factor of the electron, $\theta$ is the observation angle that results in off-axis Doppler shift. The laser intensities are denoted by the normalized vector potential:

$$
a_{0}=0.855\left(\frac{\lambda_{0}}{1 \mu \mathrm{m}}\right)\left(\frac{I}{1 \times 10^{22} \mathrm{~W} \mathrm{~m}^{-2}}\right)^{1 / 2}
$$

where $I$ is the laser intensity. The bandwidth of the spectrum is inversely proportional to the number of electron oscillations $N_{\text {osc }}$ inside the laser field: 


$$
\frac{\Delta \omega_{\mathrm{TWTS}}^{\mathrm{FWHM}}}{\omega_{\mathrm{TWTS}}}=\frac{1}{N_{\mathrm{osc}}}=\frac{d_{\mathrm{beam}}}{\lambda_{0}} \frac{1-\beta \cos \phi}{\sin \phi}
$$

where $d_{\text {beam }}$ is the diameter of the beam "used" for the TWTS process.

\section{SIMULATION RESULTS}

The simulation parameters are summarized in Table I. The relativistic electron is assumed to be initially propagating in $+x$ direction. A pulse-front-tilted laser pulse, the so-called TWTS pulse, is instantly applied to and comoves with the electron. As a result, the electron trajectory is modified by the electromagnetic field of the TWTS pulse; in addition, the electron oscillates in the $y$ direction. These small transverse oscillations lead to the $\mathrm{x}$-ray radiation emission mainly in the electron general propagation direction. The calculation of the $\mathrm{x}$-ray radiation emission is based on the electron trajectory post-processing. The electron trajectory calculation takes into account the radiation reaction process based on the Lorentz-Abraham-Dirac (LAD) model, which will be discussed in Sec. III A.

Section A, B, and C discuss TWTS for the measurement of radiation reaction, dephasing limit of a laser wakefield accelerator, and multiple electron beams in a laser wakefield accelerator, respectively.

\section{A. TWTS for the measurement of radiation reaction}

This subsection investigates the application of TWTS as an electron spectrometer to detect the radiation-reaction process. As shown in Fig. 1, another intense laser pulse, called radiation-reaction pulse, coming from the right, counterpropagates with the electron and collides with it at point zero, which is the middle of the simulation.

Understanding the radiation-reaction process is a longstanding physics challenge [22]. It stems from the backreaction on an accelerated electron from the radiation it emits. Because accurate descriptions of radiation reaction require complex calculations using quantum electrodynamics, simplified quantum models are necessary. Those simplified quantum models need testing, especially for the damping-dominated regime, which usually requires the quantum nonlinearity parameter

$$
\chi_{0}=6 \times 10^{-6} \gamma_{0} a_{0, R R}
$$

close to 1 . In the definition above, $\gamma_{0}$ is the Lorentz factor of electron before radiation reaction and $a_{0, R R}$ is the normalized vector potential of the radiation reaction pulse. A preliminary experiment based on ICS [24] toward the quantum regimes of the radiation reaction reaches $\chi_{0} \sim$ 0.07 and an energy loss $\sim 10 \%$. Another experiment [25] reaches $\chi_{0} \sim 0.2$ and an energy loss $\sim 30 \%$. However, pointing fluctuation of the high-intensity laser pulse makes its overlap with the electron beam unstable, and the RR measurements from those experiments were based on statistical analysis of many shots.

Single-shot measurements of RR are being discussed, as they remove the ambiguity of the RR measurements in the modern ICS experiments. In [26], the electron bunch is larger than the focused laser pulse and only its central part overlaps with the laser pulse and feels the radiation-reaction force. As a result, the beam's energy divergence spectrum automatically distinguishes components that do and do not experience the radiation reaction. TWTS has a potential to be alternative single-shot measurement of radiation reaction for ICS experiments. In a single shot, electrons have different energies before and after radiation reaction energy loss occurs, and this energy difference can be examined in the TWTS x-ray spectrum.

The radiation reaction model used in this paper is the Lorentz-Abraham-Dirac (LAD) model [22], which violates the principle of causality. Nonetheless, it approximates the perturbation regime of the full quantum model of radiation reaction. As shown in Eqs. (1)-(4), it is numerically straightforward to implement, as the phase space of the previous numerical step is used for the calculation of radiation force, which will be used to compute the phase space of the next numerical step.

The parameters are given in the appendix and the results are shown in Fig. 2. In (a), the radiation reaction takes
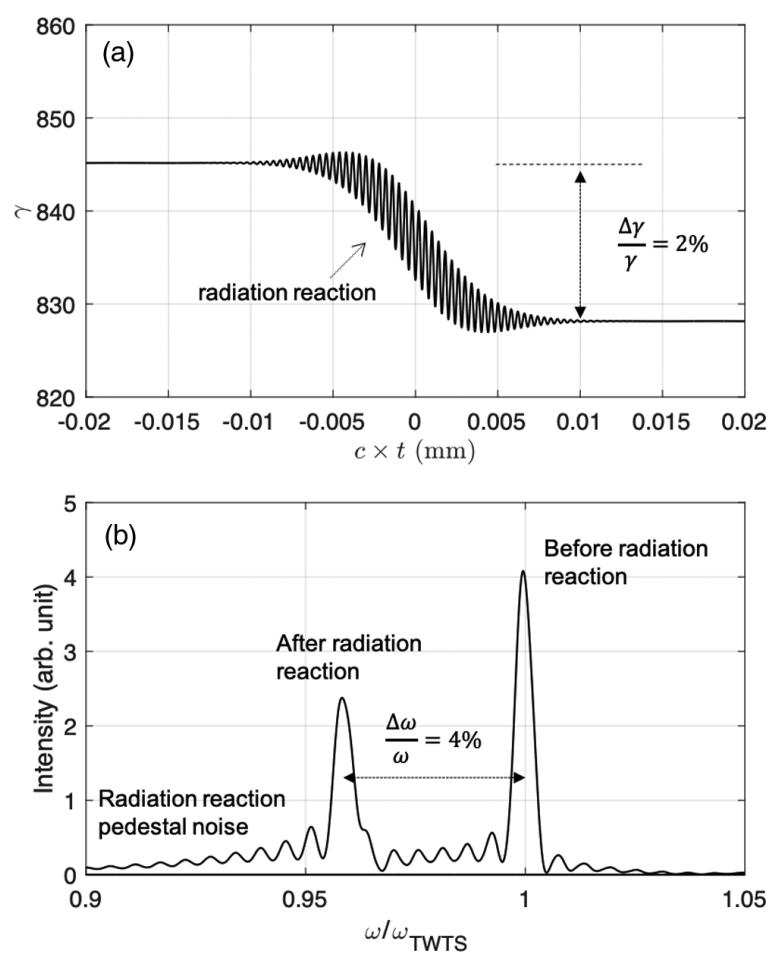

FIG. 2. A relativistic electron is continuously diagnosed with TWTS for its spectrum when it is decelerated by the radiation reaction force. (a). Electron energy drops by a certain amount due to radiation reaction friction. (b). Electron TWTS spectrum contains two peaks that correspond to energies before and after radiation reaction process. Simulation parameters can be found in Table I. 
places in the middle of the simulation and lasts for the time of about $34 \mathrm{fs}$, which is the FWHM duration of the RR laser pulse. The RR decreases the electron energy by about two percent. The X-ray wavelength shift is related to the electron energy shift, which according to Eq. (6) is about four percent, in agreement with the simulation results shown in (b).

\section{B. TWTS for the measurement of the dephasing limit of a laser wakefield accelerator}

Although laser wakefield accelerators (LWFA) have a much larger acceleration gradient $(\sim \mathrm{GV} / \mathrm{cm})$ than conventional $\mathrm{RF}$ accelerators $(\sim \mathrm{MV} / \mathrm{cm})$ [27], there are upper limits of the energy attainable from the LWFA, resulting from pump-laser depletion and electron dephasing. There have been methods proposed to overcome the pump depletion and dephasing limits: for instance, cascaded LWFA, travelingwave LWFA [28], and pulse-train LWFA [29].

To measure accurately the dephasing limit of LWFA is beneficial for understanding and sharpening the LWFA performance. The energy measured by a magnetic spectrometer for LWFA might not be the highest energy attainable from the wakefield. Instead, the detected electron bunch might leave the plasma either still in an acceleration stage or already in a deceleration (dephasing) stage. Until now, there lacks a sophisticated method to measure the dephasing limit of LWFA. This difficulty of measuring the dephasing limit can be resolved by a single-shot TWTS spectroscopy. The TWTS spectrum of an electron in a wakefield is a continuum, because the electron undergoes continuous acceleration or deceleration. Therefore, TWTS is also a method for the generation of x-ray supercontinuum, and its bandwidth can be controlled by controlling the diameter of the laser pulse, in other words, truncating the TWTS at a specific time.

A standard case of LWFA is used as a demonstrative example. In particular, based on the scaling laws for 3D nonlinear wakefield [30], a $60 \mathrm{TW}$ laser pulse is assumed to accelerate electrons up to $440 \mathrm{MeV}$ in the plasma of electron density of $n_{e}=6 \times 10^{18} \mathrm{~cm}^{-3}$. The corresponding wakefield amplitude $E_{z}[\mathrm{~V} / \mathrm{cm}]=0.96 \sqrt{n_{e}\left[\mathrm{~cm}^{-3}\right]}[27]$ within a plasma wavelength $\lambda_{p}[\mathrm{~cm}]=3.3 \times 10^{6} / \sqrt{n_{e}\left[\mathrm{~cm}^{-3}\right]}$ [27] gives approximately the wakefield amplitude slope near the center of the bubble:

$$
\frac{d \xi}{d l}\left[\mathrm{~V} / \mathrm{cm}^{2}\right]=\frac{E_{z}}{\lambda_{p}} \approx \frac{n_{e}\left[\mathrm{~cm}^{-3}\right]}{3.3 \times 10^{6}} .
$$

Compared to the typical form of the wakefield acceleration field, Eq. (10) considers only its linear part as what is justified within the bubble center. The wakefield experienced by the trapped electron is also changing in time. The electron is initially a little behind, but travels faster than, the bubble center. In the very beginning, the electron is being accelerated. Later on, as the electron reaches the bubble center the acceleration drops to zero, but the electron still moves faster than the bubble center. As the electron surpasses the bubble
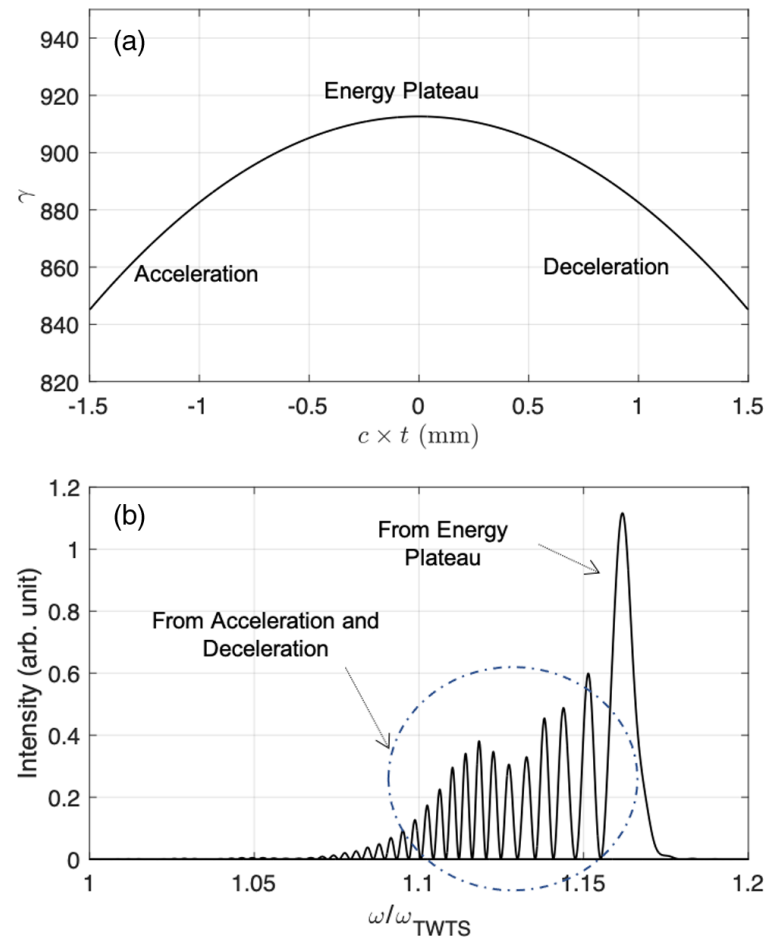

FIG. 3. A relativistic electron is continuously diagnosed with TWTS for its spectrum when it is in a laser wakefied accelerator and near the dephasing limit. (a) An electron accelerated by the wakefied to an energy plateau, before a decrease takes place due to the dephasing of the acceleration. (b) Electron TWTS spectrum contains a continuum which corresponds to the electron energy continuum. Simulation parameters can be found in Table I.

center, it experiences deceleration field and is so-called "dephased."

In Fig. 3(a), electron accelerates and decelerates due to its propagation in wakefield. Between the acceleration and deceleration stages, there is an energy plateau region, which is a result of very small acceleration or deceleration fields. Thus, the three energy stages of the electron acceleration process generate a particularly shaped TWTS x-ray supercontinuum spectrum. The acceleration and deceleration stages have lower electron energies, and therefore yield a low-energy tail in the spectrum. It should be noted here that the interference pattern for the low-energy tail is due the single-electron simulation treatment. For a practical electron beam with finite emittance, such a spectral feature does not exist because electrons emit radiation randomly with respect to each other. The energy plateau stage has the largest electron energy, which thus corresponds to the maximum $\mathrm{x}$-ray energy. In addition, the spectrum intensity is peaked near the maximum $\mathrm{x}$-ray energy, because the electron stays in the energy plateau for a relatively long time of several picoseconds. Thus, this serves as an efficient method to diagnose the dephasing limit. Moreover, assuming the symmetrical wakefield, it is possible to derive the wakefield slope from the energy evolution curve, which can be derived from the measured TWTS x-ray spectrum. 


\section{TWTS for the measurement of multiple electron beams in a laser wakefield accelerator}

A recent study [31] presents the generation of the electron beam train with the comblike energy spectrum by trapping the electrons into several following plasma wave periods. Such beam trains have potential to improve pump-probe studies considerably. A TWTS energy spectrometer has the potential to measure the temporal structure of the electron beams. By scanning the delay between the electron beams and the TWTS laser pulse, the temporal structure is mapped into the $\mathrm{x}$-ray spectrogram. This method is complementary to the original indirect method based on beam-loading, and another method based on the emitted coherent transition radiation [32,33].

To simulate the process of TWTS spectroscopy for multiple electron beams, three co-propagating relativistic electrons with different energies are separated from each other by the factor of one plasma wavelength. The first one refers to the electron that travels ahead of the other two, and the same definition for the other two. We assume the three electrons have different energies since they are injected in different bubbles: the 1st electron has an energy of $280 \mathrm{MeV}$, and the 2nd and 3rd have $240 \mathrm{MeV}$ and $220 \mathrm{MeV}$, respectively.

By scanning the delay of the TWTS laser pulse, it overlaps and comoves with different parts of the electron bunch train for a certain distance. Due to the travellingwave geometry, the respective Thomson scatterings are all narrow-band, and therefore could be distinguished from each other; although the energy differences between different electrons are very small. The spectrum can be scanned and put together, as shown in Fig. 4.

In Fig. 4, the spectral intensity is shown in logarithmic scale, as a function of x-ray photon energy and TWTS delay time. Three different delay times are used, each of which gives its own TWTS spectrum. The spectral lines shift as the delay is scanned, because the TWTS pulse overlaps with different electrons. Therefore the temporal structure of the electron array is resolved, with temporal position and energy for each electron simultaneously identified. In addition, this method is nondisruptive, and therefore can be complementary to a magnetic spectrometer [34-36].

\section{DISCUSSION}

This paper presents simulation results for a TWTS spectrometer measurement of the energy of single electrons. Several challenges need to be discussed to enhance the robustness and practicality of the method.

Traveling-wave Thomson scattering involves a spatiotemporal overlap of a laser pulse and an electron beam, with an accuracy of a few microns and a few femtoseconds. This is experimentally very challenging to achieve, due to the inherent pointing instabilities of the laser pulse, near $10 \mu \mathrm{rad}$, and that of the electron beam from laser wakefield

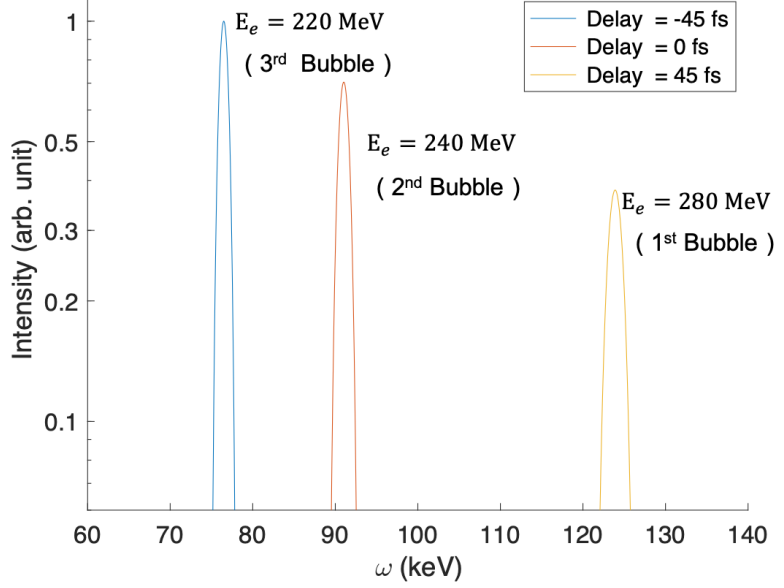

FIG. 4. TWTS x-ray spectrogram for investigating the temporal structure of the electron beam bunches. Here three electrons, representing three electron beams in respective wakefield bubbles, are diagnosed with the delay-scanning TWTS. Three timeresolved spectral lines corresponds to respective energies of electrons in different bubbles.

accelerator, near $1 \mathrm{mrad}$. The electron beam jitters much more than the laser beam, and needs to be stabilized, although not much effort has been made to reduce the pointing instability of LWFA electron beams. A common method to increase the frequency of occurrence of overlap is to overlap near the exit of the LWFA, so that the spatial mismatch is reduced due to limited propagation distance of the electron beam. Nevertheless, such overlap is still not that stable, and usually statistical analysis is required. More work on stabilizing the pointing of the LWFA electron beams is required.

Our simulations are limited to the dynamics of an electron in a plane geometry. Although this simplified geometry grasps the basic physics and insights of the TWTS as an electron spectrometer, it nevertheless neglects macroscopic effects and phase-space effects. For example, in multi-electron beams, finite electron-beam emittance broadens the Thomson $\mathrm{x}$-ray spectrum:

$$
\begin{aligned}
\left(\frac{\Delta \omega_{\text {TWTS }}}{\omega_{\text {TWTS }}}\right)^{2}= & \left(\frac{2 \Delta \gamma}{\gamma}\right)^{2}+\left(\gamma^{2} \sigma_{r}^{2}\right)^{2}+\left(\frac{2 \Delta \omega_{0}}{\omega_{0}}\right)^{2} \\
& +\frac{1}{N_{\text {osc }}^{2}}+\left(\gamma^{2} \theta^{2}\right)^{2}
\end{aligned}
$$

where $\Delta \gamma$ is the energy spread of the electron beam, $\sigma_{r}$ is the electron angular spread and $\Delta \omega_{0}$ is the laser bandwidth. Therefore, for applications requiring high spectral resolution, it is necessary to reduce unwanted broadening effect as much as possible. The electron beam needs to have very small energy spread and divergence, such as that has been demonstrated [9], in order not to smear-out specific spectral lines of TWTS for measurements of radiation reaction and diagnostics of multi-bubble injection LWFA. More 
specifically, a divergence of $0.4 \mathrm{mrad}$ for a $400-\mathrm{MeV}$ electron beam induces a natural energy spread of $10 \%$ in its TWTS spectrum. From an experimental point of view, such a spectral broadening effect limits its accuracy. As a result, when TWTS of such a electron beam is used for the measurement of radiation reaction, according to Eq. (6), the minimum measurable deceleration amount is around 5\%. Thus, the simulated results presented in Fig. 2 are currently not achievable in practice, and further improvement in the electron beam emittance is needed.

The free spectral range of the TWTS spectrometer is very large. As shown in Eq. (8), the x-ray energy is dependent on the crossing angle between the electron and laser pulse propagation. As a result, any range of electron energy can be mapped into x-rays with different scales, by changing the crossing angle. The pulse-front tilt-angle can be tuned correspondingly to maintain the traveling-wave structure. For convenience, small crossing angles can be used to map the (ultra-)relativistic electron beams into, for example, EUV or soft x-rays, which can be measured by many commercial x-ray spectrometers.

Another important parameter of a spectrometer is the noise level, while the method to increase of signal-to-noise ratio depends on the process itself. For example, the radiation reaction process not only decelerates electrons in the propagation direction, but also induces large-amplitude electron oscillations perpendicular to the propagation, which generate intense and red-shifted Thomson x-rays in the electron propagation direction, which might overlap with the main TWTS signal. In other words, the RR process may itself hinder measurement by the TWTS spectrometer. Nevertheless, the TWTS signal can be increased to overwhelm the RR noise, by either increasing the TWTS laser

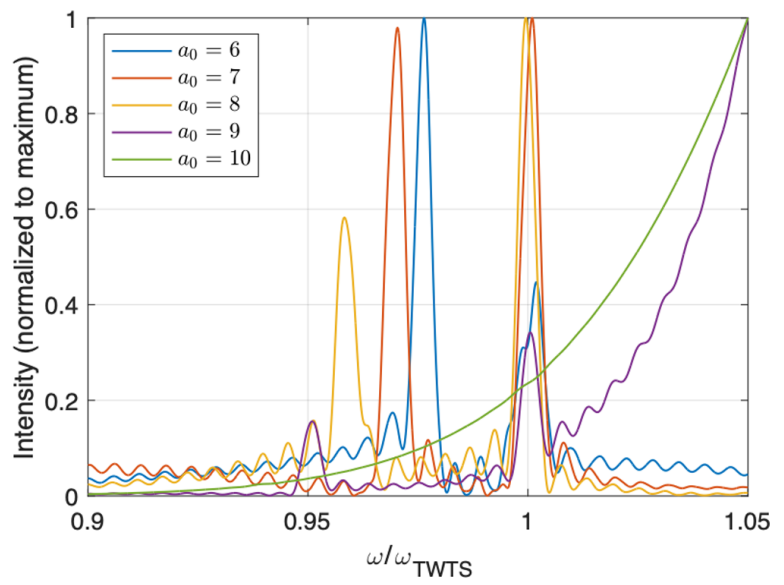

FIG. 5. Noise analysis for the simulations presented in Fig. 2(b). The field strength of the radiation reaction pulse is varied here to see the changes in noise for x-ray spectrum from the radiaiton reaction process. For $a_{0}=10$ the TWTS signals are dominated by the noise. To increase the TWTS signals, either a stronger TWTS laser pulse or a longer TWTS interaction length is needed. strength or the traveling-wave distance. We did the former for our simulations to save the simulation time. As an example, for the investigation of RR noise, we started from the simulations presented in Fig. 2 and varied the strength of the radiation reaction pulse to see the change in the noise level. The results and analysis are presented in Fig. 5, where case $a_{0}=8$ is identical to that in Fig. 2(b). With increasing $a_{0}$, the radiation reaction noise becomes larger and larger. For $a_{0}=9$, the main two TWTS peaks sitting on the RR noise are only marginally apparent, while for $a_{0}=10$, the RR noise dominates the TWTS signals. Thus, either a stronger TWTS pulse or a longer TWTS distance is needed to increase the TWTS signal amplitudes such that they can emerge from the RR noise.

\section{SUMMARY}

In summary, traveling-wave Thomson scattering is proposed as a high-resolution spectrometer for the spatiotemporal energy measurement of relativistic electron beams. The TWTS spectrometer is applicable to many laser-based experiments. For example, the long-standing problem of radiation reaction can be unambiguously measured by TWTS in a single shot, and thereby enable quantitative examination of fundamental nonlinear QED theory. For another example, electron acceleration and deceleration in a wakefield can be probed by TWTS, with the dephasing limit accurately measured and the laserwakefield amplitude determined. The broadened TWTS xray spectrum, due to the continuous electron acceleration and deceleration, is also a method for the generation of controlled x-ray supercontinuum. Last, multibucket electron injection can also be temporally diagnosed with TWTS, which helps to differentiate between different injection mechanisms and improve electron-injection control. The proposed single-electon method calls for macroscopic studies to fully picture the physics involved in the full phase space. In addition, future studies of travellingwave Thomson scattering require reduced electron-beam emittance and stabilization of electron-beam pointing.

\section{ACKNOWLEDGMENTS}

This material is based upon work supported by the US Department of Energy, High-Energy Physics (HEP), under Award No. DE-SC0019421 (Controlled Injection of electrons for the Improved Performance of Laser-Wakefield Acceleration); the U.S. Department of Energy and National Nuclear Security Administration (NNSA), under Award No. DE-SC0019311 (Laboratory Study of Nonlinear QED in intense Laser-Matter Interactions); the European Research Council (ERC-2014-CoG Grant No. 647121). This support does not constitute an express or implied endorsement on the part of the Government. We gratefully acknowledge valuable contributions from C. Fruhling, D. Haden, J. Wang and S. Banerjee. 


\section{APPENDIX: SIMULATION PARAMETERS}

This Appendix provides a detailed table of simulation parameters for respective results presented in Sec. III of the manuscript. The last three columns of the table respectively give parameters for the simulation of "TWTS for the measurement of radiation reaction," simulation of "TWTS for the measurement of the dephasing limit of a laser wakefield accelerator," and simulation "TWTS for the measurement of multiple electron beams in a laser wakefield accelerator."

TABLE I. Parameters for the simulations.

\begin{tabular}{lcccc}
\hline \hline Parameters & Symbol & Value $($ Section A) & Value (Section B) & Value (Section C) \\
\hline TWTS laser PFT angle & $\alpha_{0, \mathrm{TWTS}}$ & $15^{\circ}$ & $15^{\circ}$ & $15^{\circ}$ \\
TWTS overlap distance & $l_{0, \mathrm{TWTS}}$ & $2.4 \mathrm{~mm}$ & $3 \mathrm{~mm}$ & $0.6 \mathrm{~mm}$ \\
TWTS laser wavelength & $\lambda_{0, \mathrm{TWTS}}$ & $0.8 \mu \mathrm{m}$ & $0.8 \mu \mathrm{m}$ & $0.8 \mu \mathrm{m}$ \\
TWTS laser strength & $a_{0, \mathrm{TWTS}}$ & $1 \times 10^{-3}$ & $1 \times 10^{-5}$ & $1 \times 10^{-5}$ \\
TWTS laser FWHM duration & $\tau_{0, \mathrm{TWTS}}$ & $34 \mathrm{fs}$ & $34 \mathrm{fs}$ & $34 \mathrm{fs}$ \\
TWTS laser waist & $d_{0, \mathrm{TWTS}}$ & $1 \mathrm{~mm}$ & $1 \mathrm{~mm}$ & $\ldots$ \\
RR laser wavelength & $\lambda_{0, \mathrm{RR}}$ & $0.8 \mu \mathrm{m}$ & $\ldots$ & $\ldots$ \\
RR laser strength & $a_{0, \mathrm{RR}}$ & 8 & $\ldots$ & $\ldots$ \\
RR laser FWHM duration & $\tau_{0, \mathrm{RR}}$ & $34 \mathrm{fs}$ & $\ldots$ & $\ldots$ \\
RR laser waist & $d_{0, \mathrm{RR}}$ & $2 \mu \mathrm{m}$ & $432 \mathrm{MeV}$ & $280 \mathrm{MeV}$ \\
1st electron energy & $E_{0,1}$ & $432 \mathrm{MeV}$ & $\ldots$ & $240 \mathrm{MeV}$ \\
2nd electron energy & $E_{0,2}$ & $\ldots$ & $\ldots$ & $220 \mathrm{MeV}$ \\
3rd electron energy & $E_{0,3}$ & $\ldots$ & $0 \mu \mathrm{m}$ & $13.5 \mu \mathrm{m}$ \\
1st electron position & $x_{0,1}$ & $0 \mu \mathrm{m}$ & $\ldots$ & $0 \mu \mathrm{m}$ \\
2nd electron position & $x_{0,2}$ & $\ldots$ & $\ldots$ & $-13.5 \mu \mathrm{m}$ \\
3rd electron position & $x_{0,3}$ & $\ldots$ & $\ldots$ & $\ldots$ \\
\hline \hline
\end{tabular}

[1] E. Esarey, P. Sprangle, A. Ting, and S. Ride, Laser synchrotron radiation as a compact source of tunable, short pulse hard x-rays, Nucl. Instrum. Methods Phys. Res., Sect. A 331, 545 (1993).

[2] I. Andriyash, E. d'Humieres, V. Tikhonchuk, and P. Balcou, X-Ray Amplification from a Raman Free-Electron Laser, Phys. Rev. Lett. 109, 244802 (2012).

[3] H. Kotaki, M. Kando, H. Dewa, S. Kondo, T. Watanabe, T. Ueda, K. Kinoshita, K. Yoshii, M. Uesaka, and K. Nakajima, Compact X-ray sources by intense laser interactions with beams and plasmas, Nucl. Instrum. Methods Phys. Res., Sect. A 455, 166 (2000).

[4] N. D. Powers, I. Ghebregziabher, G. Golovin, C. Liu, S. Chen, S. Banerjee, J. Zhang, and D. P. Umstadter, Quasimonoenergetic and tunable x-rays from a laser-driven Compton light source, Nat. Photonics 8, 28 (2014).

[5] K. Khrennikov, J. Wenz, A. Buck, J. Xu, M. Heigoldt, L. Veisz, and S. Karsch, Tunable All-Optical QuasiMonochromatic Thomson x-Ray Source in the Nonlinear Regime, Phys. Rev. Lett. 114, 195003 (2015).

[6] G. Golovin, S. Banerjee, C. Liu, S. Chen, J. Zhang, B. Zhao, P. Zhang, M. Veale, M. Wilson, P. Seller et al., Intrinsic beam emittance of laser-accelerated electrons measured by $\mathrm{x}$-ray spectroscopic imaging, Sci. Rep. 6, 24622 (2016).
[7] J. Krämer, A. Jochmann, M. Budde, M. Bussmann, J. Couperus, T. Cowan, A. Debus, A. Köhler, M. Kuntzsch, A. L. García et al., Making spectral shape measurements in inverse Compton scattering a tool for advanced diagnostic applications, Sci. Rep. 8, 1398 (2018).

[8] Z. Qin, C. Yu, W. Wang, J. Liu, W. Li, R. Qi, Z. Zhang, J. Liu, M. Fang, K. Feng et al., Ultralow-emittance measurement of high-quality electron beams from a laser wakefield accelerator, Phys. Plasmas 25, 023106 (2018).

[9] W. Wang, W. Li, J. Liu, Z. Zhang, R. Qi, C. Yu, J. Liu, M. Fang, Z. Qin, C. Wang et al., High-Brightness HighEnergy Electron Beams from a Laser Wakefield Accelerator Via Energy Chirp Control, Phys. Rev. Lett. 117, 124801 (2016).

[10] C. Tang, W. Huang, R. Li, Y. Du, L. Yan, J. Shi, Q. Du, P. Yu, H. Chen, T. Du et al., Tsinghua Thomson scattering Xray source, Nucl. Instrum. Methods Phys. Res., Sect. A 608, S70 (2009).

[11] S. Rykovanov, C. Geddes, J. Vay, C. Schroeder, E. Esarey, and W. Leemans, Quasi-monoenergetic femtosecond photon sources from Thomson Scattering using laser plasma accelerators and plasma channels, J. Phys. B 47, 234013 (2014).

[12] A. Debus, M. Bussmann, M. Siebold, A. Jochmann, U. Schramm, T. Cowan, and R. Sauerbrey, Traveling-wave Thomson scattering and optical undulators for high-yield EUV and X-ray sources, Appl. Phys. B 100, 61 (2010). 
[13] C. Chang, C. Tang, and J. Wu, High-Gain ThompsonScattering x-Ray Free-Electron Laser by Time-Synchronic Laterally Tilted Optical Wave, Phys. Rev. Lett. 110, 064802 (2013).

[14] K. Steiniger, D. Albach, M. Bussmann, M. Loeser, R. Pausch, F. Röser, U. Schramm, M. Siebold, and A. Debus, Building an optical free-electron laser in the TravelingWave Thomson-Scattering geometry, Front. Phys. 6, 155 (2019), https://www.frontiersin.org/articles/10.3389/fphy .2018.00155/full.

[15] J. Hebling, G. Almasi, and I. Z. Kozma, and J. Kuhl, Velocity matching by pulse front tilting for large-area Thzpulse generation, Opt. Express 10, 1161 (2002).

[16] P. Baum and A. H. Zewail, Breaking resolution limits in ultrafast electron diffraction and microscopy, Proc. Natl. Acad. Sci. U.S.A. 103, 16105 (2006).

[17] J.-C. Chanteloup, E. Salmon, C. Sauteret, A. Migus, P. Zeitoun, A. Klisnick, A. Carillon, S. Hubert, D. Ros, P. Nickles et al., Pulse-front control of 15-TW pulses with a tilted compressor, and application to the subpicosecond traveling-wave pumping of a soft-X-ray laser, J. Opt. Soc. Am. B 17, 151 (2000).

[18] R. King, G. Pert, S. McCabe, P. Simms, A. MacPhee, C. Lewis, R. Keenan, R. O'Rourke, G. Tallents, S. Pestehe et al., Saturated x-ray lasers at 196 and $73 \AA$ pumped by a picosecond traveling-wave excitation, Phys. Rev. A 64, 053810 (2001).

[19] M. Grünig, C. Imesch, F. Staub, and J. E. Balmer, Saturated $\mathrm{x}$-ray lasing in Ni-like $\mathrm{Sn}$ at $11.9 \mathrm{~nm}$ using the GRIP scheme, Opt. Commun. 282, 267 (2009).

[20] Q. Chen, Z. Wu, A. Morozov, and S. Suckewer, Stimulated Raman near-backscattering with a pulse-front-tilted pump, Phys. Plasmas 25, 093110 (2018).

[21] Z. Wu, Q. Chen, A. Morozov, and S. Suckewer, Compression of laser pulses by near-forward Raman amplification in plasma, Phys. Plasmas 27, 013104 (2020).

[22] L. D. Landau, The Classical Theory of Fields (Elsevier, New York, 2013), Vol. 2.

[23] V. Horný, J. Nejdl, M. Kozlová, M. Krůs, K. Boháček, V. Petržílka, and O. Klimo, Temporal profile of Betatron radiation from laser-driven electron accelerators, Phys. Plasmas 24, 063107 (2017).

[24] J. Cole, K. Behm, E. Gerstmayr, T. Blackburn, J. Wood, C. Baird, M. J. Duff, C. Harvey, A. Ilderton, A. Joglekar et al., Experimental Evidence of Radiation Reaction in the Collision of a High-Intensity Laser Pulse with a LaserWakefield Accelerated Electron Beam, Phys. Rev. X 8, 011020 (2018).

[25] K. Poder, M. Tamburini, G. Sarri, A. Di Piazza, S. Kuschel, C. Baird, K. Behm, S. Bohlen, J. Cole, D. Corvan et al., Experimental Signatures of the Quantum Nature of Radi- ation Reaction in the Field of an Ultraintense Laser, Phys. Rev. X 8, 031004 (2018).

[26] C. D. Baird, C. D. Murphy, T. Blackburn, A. Ilderton, S. Mangles, M. Marklund, and C.P. Ridgers, Realising single-shot measurements of quantum radiation reaction in high-intensity lasers, New J. Phys. 21, 053030 (2019).

[27] E. Esarey, C. Schroeder, and W. Leemans, Physics of laserdriven plasma-based electron accelerators, Rev. Mod. Phys. 81, 1229 (2009).

[28] A. Debus, R. Pausch, A. Huebl, K. Steiniger, R. Widera, T. E. Cowan, U. Schramm, and M. Bussmann, Circumventing the Dephasing and Depletion Limits of Laser-Wakefield Acceleration, Phys. Rev. X 9, 031044 (2019).

[29] P. Tomassini, S. De Nicola, L. Labate, P. Londrillo, R. Fedele, D. Terzani, and L. A. Gizzi, The resonant multipulse ionization injection, Phys. Plasmas 24, 103120 (2017).

[30] W. Lu, M. Tzoufras, C. Joshi, F. Tsung, W. Mori, J. Vieira, R. Fonseca, and L. Silva, Generating multi-GeV electron bunches using single stage laser wakefield acceleration in a 3D nonlinear regime, Phys. Rev. Accel. Beams 10, 061301 (2007).

[31] G. Golovin, V. Horný, W. Yan, C. Fruhling, D. Haden, J. Wang, S. Banerjee, and D. Umstadter, Generation of ultrafast electron bunch trains via trapping into multiple periods of plasma wakefields, Phys. Plasmas 27, 033105 (2020).

[32] O. Lundh, C. Rechatin, J. Lim, V. Malka, and J. Faure, Experimental Measurements of Electron-Bunch Trains in a Laser-Plasma Accelerator, Phys. Rev. Lett. 110, 065005 (2013).

[33] M. Heigoldt, A. Popp, K. Khrennikov, J. Wenz, S.-W. Chou, S. Karsch, S. Bajlekov, S. Hooker, and B. Schmidt, Temporal evolution of longitudinal bunch profile in a laser wakefield accelerator, Phys. Rev. Accel. Beams 18, 121302 (2015).

[34] Y. Glinec, J. Faure, A. Guemnie-Tafo, V. Malka, H. Monard, J. Larbre, V. De Waele, J. Marignier, and M. Mostafavi, Absolute calibration for a broad range single shot electron spectrometer, Rev. Sci. Instrum. 77, 103301 (2006).

[35] K. Nakamura, W. Wan, N. Ybarrolaza, D. Syversrud, J. Wallig, and W. Leemans, Broadband single-shot electron spectrometer for $\mathrm{GeV}$-class laser-plasma-based accelerators, Rev. Sci. Instrum. 79, 053301 (2008).

[36] C. M. Sears, S. B. Cuevas, U. Schramm, K. Schmid, A. Buck, D. Habs, F. Krausz, and L. Veisz, A high resolution, broad energy acceptance spectrometer for laser wakefield acceleration experiments, Rev. Sci. Instrum. 81, 073304 (2010). 